\title{
The self-regulation of hypothalamic rewarding brain stimulus after innoculation of Mycobacterium butyricum inducing chronic arthritis
}

\author{
PH. DE WITTE \\ Université Catholique de Louvain, Louvain-la-Neuve, Belgium \\ F. COLPAERT \\ Janssen Pharmaceutica, Beerse, Belgium \\ and \\ P. SCHMITT \\ Centre de Neurochimie du C.N.R.S., Strasbourg, France
}

\begin{abstract}
Rats exhibiting steady self-stimulation behavior at the posterolateral area of the hypothalamus were allowed to self-regulate the duration of the brain stimulus after inoculation of $M y$ cobacterium butyricum inducing long-lasting adjuvant arthritis. Results show that the barpressing rate as well as the total time spent in self-stimulation increase in parallel with the development of arthritis, as estimated by the diameter of the hind limbs. Several hypotheses are presented which attempt to explain the neural mechanism underlying the compensatory behavior of increasing self-administration of rewarding electrical brain stimuli during chronic pain.
\end{abstract}

Activation of the rewarding brain system can attenuate the negative effect induced by an external aversive situation (Black, Cianci, \& Markowitz, 1972; Brady \& Conrad, 1960; Cox \& Valenstein, 1965). According to these authors, an aversive situation usually avoided by animals could be better tolerated when a positive affective state is created by direct brain stimulation, inducing a powerful reward. Experiments showing acceptance by animals of longer aversive footshock periods during self-stimulation strengthen this hypothesis (De Witte, 1979).

The experiments that show the action of the compensatory effect given by direct brain stimulation are based on external and acute stimuli (peripheral footshock or external natural aversive situation). However, in these paradigms, pain stimuli remain transient and the compensation ensured by rewarding brain stimulation may be largely different under continuous and chronic pain stimuli. Results obtained by introducing electrodes in the periaqueductal gray matter showed a strong relationship between medium long-lasting pain (using the Formalin pain test) and

This work was supported by a training grant offered by the European Training Program. Ph. De Witte's mailing address is: Université Catholique de Louvain, Laboratoire de Psychobiologie, Tour De Serres, 1, Place Croix du Sud, 1348 Louvain-la-Neuve, Belgium. F. Colpaert and P. Schmitt are associated with the Department of Pharmacology (Janssen) and the Laboratoire de Neurophysiologie (C.N.R.S.), respectively. the current threshold for barpressing for brain stimulation (Dennis, Choiniere, \& Melzack, 1980). Nevertheless, in this last experiment, injection of Formalin never induced a chronic pain state per se. Secondly, these brain stimulations induce both analgesia and self-stimulation.

Our experiment attempted to test this self-induced brain compensatory balance with a long-lasting painful stimulus (Mycobacterium butyricum) that inflicted chronic adjuvant arthritis. It had been suggested that inoculation of rats with an arthritogenic mycobacterial product (Colpaert, De Witte, Maroli, Awouters, Niemegeers, \& Janssen, 1980) might produce a valid paradigm of chronic pain that would persist for weeks, to present its highest painful peak in Week 3 and to terminate in Week 8 after inoculation. We assumed, therefore, that the animals would consistently increase their self-stimulation as the arthritis developed. These brain stimulations never produced analgesia as had periaqueductal gray stimulation.

\section{METHOD}

\section{Subjects, Electrodes, and Histology}

Male albino rats of the Wistar strain, weighing approximately $300 \mathrm{~g}$ at the time of operation, were implanted with a monopolar nickel-chrome electrode $(.25 \mathrm{~mm})$ insulated except for the cross section of the tip. The electrodes were implanted stereotaxically according to the following coordinates: $\mathrm{A}, 3.5 \mathrm{~mm}$ behind bregma; L, $1.2 \mathrm{~mm}$; H, $8.3 \mathrm{~mm}$ below the skull surface (lateral posterior 
hypothalamus). The indifferent electrode was placed $2 \mathrm{~mm}$ in front of the bregma.

The animals were allowed 1 week to recover and then were trained to self-stimulate. Upon completion of the experiment, the subjects were killed and the brains removed and placed in 10\% Formalin in saline for 10 days. Then the brains were frozen and cut at $100 \mu$; the sections were stained with cresyl violet.

The animals were allowed to self-stimulate in modified Skinner boxes using brain stimulations of 0.2 -sec-train duration and 0.2 msec pulse, delivered at a frequency of $100 \mathrm{~Hz}$. The current intensity varied between 60 and $200 \mu \mathrm{A}$ to adjust equal performance from rat to rat with a mean of $150 \mu \mathrm{A}$. An experimental group of 18 rats, presenting consistent and stable self-stimulation, was formed.

\section{Apparatus}

Rats exhibiting consistent self-stimulation behavior were tested in Plexiglas enclosures that were $45 \mathrm{~cm}$ long, $30 \mathrm{~cm}$ wide, and $60 \mathrm{~cm}$ high. A lever $(4 \times 3 \times 0.5 \mathrm{~cm})$ was placed $2 \mathrm{~cm}$ above the floor. A pressure of $40 \mathrm{~g}$ upon the lever produced brain stimulation for as long as the lever was depressed. The duration of the leverpress was automatically recorded.

\section{Procedure}

The sample of 18 rats was allowed to self-regulate its intracranial stimulation. Two electrical combinations were chosen: (1) a 0.1-msec pulse width delivered at a frequency of $100 \mathrm{~Hz}$ and (2) $0.2-\mathrm{msec}$ pulse width delivered at $200 \mathrm{~Hz}$. The second combination induced a high degree of reinforcement, whereas the first induced a lower one (De Witte, 1979). The current intensity remained constant at the self-stimulation behavior threshold, with a mean of $150 \mu \mathrm{A}$ for the sample. During a daily morning session, the animals were allowed to self-regulate the first electrical combination for a 10-min period and the second for another 10-min period.

During each period, the animals depressed or released the lever that delivered or relieved the brain stimulation.

After 3 weeks of daily self-regulation of brain stimulations, during which the performance of self-stimulation remained stable, all trial performances for these 3 weeks were averaged, as shown for control preinjection self-stimulation performance in Figure 2. At this point, the animals were randomly divided into two groups. The first group of 11 animals was inoculated with Mycobacterium butyricum (the precise procedure is described elsewhere; Awouters, Niemegeers, Lenaerts, \& Janssen, 1975). Each animal of this experimental sample was intradermally inoculated in its tail base with $0.05 \mathrm{ml}$ of a suspension containing heat-killed $M y$ cobacterium butyricum suspended in paraffin oil $(5 \mathrm{mg} / \mathrm{ml})$. The second group, that is, the remaining 7 rats, received $0.05 \mathrm{ml}$ of the vehicle (i.e., paraffin oil) by the same route. These animals were used as a control group. After 3 days, animals of both groups were tested for self-stimulation once a day for 10 weeks (no tests during the weekend).

On the 5th day of each week, the diameters of the paws of the rats in the experimental and control groups were measured and the means recorded in millimeters.

The effects of the Mycobacterium butyricum and the vehicle inoculations upon the self-regulation of rewarding brain stimulation were estimated as follows: For each period of self-stimulation, the rewarding value induced by each electrical brain stimulation was calculated on the basis of the total time the animal had selfstimulated (T) and the number of barpresses $(N)$. The value of $T$ and $\mathbf{N}$ of the experimental group was calculated as a percentage of the value of $\mathrm{T}$ and $\mathrm{N}$ of the control group, during the same week after the injection. For example, the values of the experimental group's $\mathrm{T}$ and $\mathrm{N}$ at Week 3 after inoculation were compared with the control group's $\mathrm{T}$ and $\mathrm{N}$ at Week 3 after the vehicle injection.

\section{RESULTS}

Relationships Between the Estimation of the Intracranial Reward, i.e., $T$ and $N$, After Vehicle Injection

Figure 1 shows the mean performances of the vehicle-injected sample in $\mathrm{T}$ and $\mathrm{N}$ for both electrical parameter combinations of the brain stimulus. Each value represents the mean performance for the seven rats over a week. The Bravais-Pearson correlation coefficients which relate these performances showed that $\mathrm{T}$ was strongly positively correlated with $\mathrm{N}$ (.64 and .63 , respectively, for both combinations of electrical parameters). In other words, changes in the rate of self-regulated barpressing by animals having received the vehicle injection were associated with the same changes in the total time spent self-stimulating.

Using the Friedman test, there were observable differences between the rate of barpressing before and after vehicle injection. The rate performance decreased for both brain-stimulation combinations after the injection, whereas $T$ remained at the baseline level. Nevertheless, no differences occurred between performances for the remaining 9 weeks.

As regards the paw diameter, it remained unchanged at $7.5 \mathrm{~mm}$ throughout the experiment.

\section{Relationships Between the Effects of $M y c o$ - bacterium butyricum and the Intracranial Reward as Estimated by $T$ and $N$}

Figure 2 indicates the time-course of the changes in the diameter of the hindlimbs. This represented a valid criterion for estimating the progressive development of adjuvant arthritis (Colpaert et al., 1980).

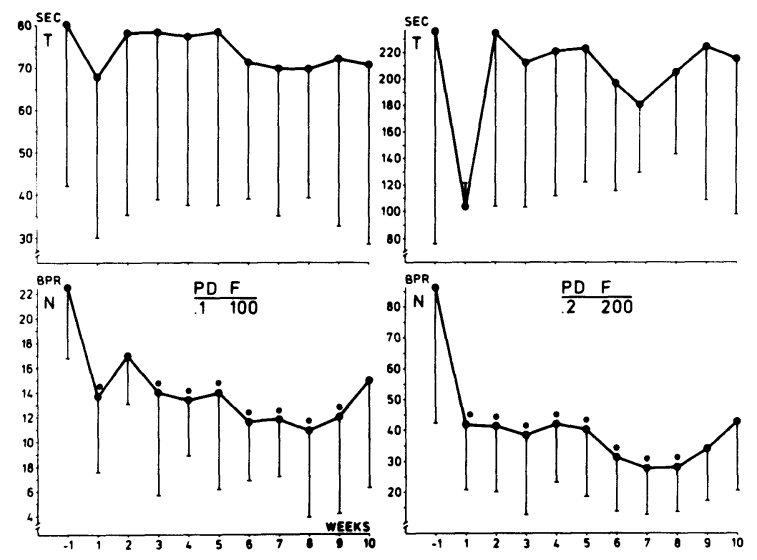

Figure 1. The mean values in $\mathbf{T}$ and $\mathbf{N}$ for both electrical brain stimulations for the control group. The total possible time was 600 sec. The asterisk near the values indicates the statistical difference from the week before the oil injection, using the Friedman test $(p<.05)$. 


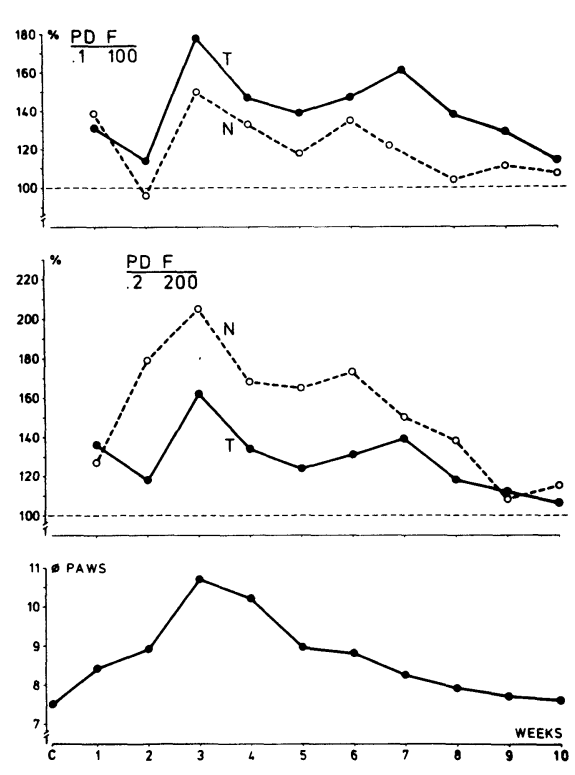

Figure 2. These figures represent the mean percentage of performance, as compared with vehicle performance, obtained during the self-regulation of a rewarding brain stimulation $(0.1 \mathrm{msec} /$ $100 \mathrm{~Hz}$ ) and a high rewarding stimulus $(0.2 / 200)$. The number of barpresses (N) and the total time spent in self-stimulation (T) are ordered as a function of the sequence of weeks. The two upper figures show a shape parallel to the modification of the diameter of the hindpaws (bottom figure). In other words, an increase in the paw diameter, as an index of the pain induced by the Mycobacterium butyricum, is accompanied by an increase in the selfregulation of the rewarding brain stimuli.

As shown earlier, in the experimental group these diameters increased to their peak during the 3rd week and then decreased asymptomatically to a level which remained above that of the control group until Week 9.

With regard to self-regulation of the rewarding brain stimulations during adjuvant arthritis, Figure 2 shows that the experimental group increased the total duration of effective brain stimulation as well as the rate of barpressing for rewarding brain stimulation. The Bravais-Pearson coefficient of correlation (Table 1) between the increase of $T$ and $N$ and the parallel increase of the diameter of the hindlimbs clearly shows that animals developing adjuvant arthritis increase proportionately their brain intake of rewarding electrical stimulation. Figure 1 and Table 1 also show the positive relationships between the rate of self-stimulation $(\mathrm{N})$ and the total time of selfstimulation (T). It thus appears that the effect of the injection of Mycobacterium butyricum on the selfregulated rewarding brain stimulations results in an increase of the rate of barpressing and of the total time spent self-stimulating rewarding electrical combinations. This increase was much more pronounced when the higher rewarding brain stimulation was used (i.e., 0.2-200).

\section{DISCUSSION}

The effects of the injection of Mycobacterium butyricum upon the level of self-stimulated behavior are to induce an increase in the rate of barpressing and in the total duration of brain self-stimulation. One could suggest that animals injected with a compound producing adjuvant arthritis in the hindlimbs would continue to press the bar longer, because they could not easily move their hindquarters. An extension of this interpretation would show a parallel decrease in the rate of barpressing (N). As Figure 1 shows, the normal pattern of behavior after an oil paraffin injection, which never developed swollen limbs, was a decrease in the frequency of barpressing (N) while the time spent in self-stimulation (T) remained unchanged. However, after the inoculation, animals always increased both the rate and the time spent in self-stimulation.

Since Ball (1957) showed that stimulation of the central rewarding pathway is able to block the input of painful signals at the trigeminal nerve level, the opening of a general gate system directly activated by such stimulation is postulated. Indeed, further experiments showed that rewarding brain stimulations act as an inhibition of the afferent system. It is now clear that activation of a particular neural brain network could have an inhibiting effect on sensory transmission through a spinal cord gate (McIntire, 1966). In this way, the rewarding property of such brain stimulation could be a secondary characteristic. It seems that such brain sites, which induce both analgesia and rewarding effects, are sites at which overlaps exist between the two systems. Some authors have found a direct relationship between the level of analgesia induced and the level of self-stimulation (Dennis et al., 1980; Liebman, Mayer, \& Liebeskind, 1973). Yet, the different refractory periods found in the two different neural populations underlying analgesia and self-stimulation in the lateral part of the hypothalamus (Rose, 1974) and the pharmacological

Table 1

Bravais-Pearson Correlations Between Number, Time, and Diameter of Hindlimbs After Inoculation of the Mycobacterium Butyricum

\begin{tabular}{cccc}
\hline \multirow{2}{*}{$\begin{array}{c}\text { Stimulation } \\
\text { Condition }\end{array}$} & $\begin{array}{c}\text { Correlation } \\
\text { with Paw } \\
\text { Diameter }\end{array}$ & $\begin{array}{c}\text { Time and } \\
\text { Number } \\
\text { Correlation }\end{array}$ \\
\hline \multirow{3}{*}{ Low } & Time & .63 & .74 \\
& No. & .65 & \\
High & Time & .77 & .72 \\
\hline
\end{tabular}

Note - Low $=0.1 \mathrm{msec} / 100 \mathrm{~Hz} ;$ high $=0.2 \mathrm{msec} / 200 \mathrm{~Hz}$. Time $=$ total duration of self-administered stimulation; No. = total number of barpresses. 
distinction between these two systems (Stark \& Fuller, 1972) support the idea of well-differentiated painsuppressive and rewarding systems.

With respect to the pain-suppressive system, the direct excitation produced by brain stimulation that induces analgesia of definite sites in the midbrain, such as the central gray matter, might be reversible by naloxone, a narcotic antagonist (Akil, Mayer, \& Liebeskind, 1975), even though the release of endogenous opioid peptides occurs during acute stress (Akil, Madden, Patrick, \& Barchas, 1976; Chance, White, Krynock, \& Rosecrans, 1977). From another source, facilitation of self-stimulation has been reported following the administration of various exogenous opiates, and this has been interpreted as being a consequence of the reinforcing value of these drugs (Adams, Lorens, \& Mitchell, 1972; Esposito \& Kornetsky, 1977; Maroli, Tsang, \& Stutz, 1978). It seems, therefore, that the release of endorphinergic peptides after Mycobacterium butyricum could increase the effectiveness of ICSS by activating, as Goldstein (1976) did, an opioid system which remained passive until becoming tonically active during painful stress.

The alternative interpretation was to grant the primary property to the positive arousal obtained via rewarding brain stimulation. While the posterolateral area of the hypothalamus is not traditionally linked with classic pain pathways, it has often been proposed that this limbic structure controls affective components of pain (Melzack \& Wall, 1965). We thus assume that the animal reduces the negative effect of the chronic arthritis by increasing the duration of the positive arousal through rewarding brain stimulation. It seems, therefore, that rats compensate for the long-lasting negative property produced by the injection of Mycobacterium butyricum by an increase in the level of excitation of the self-stimulation neural pathway.

\section{REFERENCES}

Adams, W. J., Lorens, S. A., \& Mitchell, C. L. Morphine enhances lateral hypothalamic self-stimulation in the rat. Proceedings of the Society for Experimental Biology and Medicine, 1972, 140, 770-771.

Akil, H., Madden, J., Patrick, R. L., \& Barchas, J. D. Stress-induced increase in endogenous opiate peptides: Concurrent analgesia and its partial reversal by naloxone. In $\mathbf{H}$. W. Kosterlitz (Ed.), Opiates and endogenous opioid peptides. Amsterdam: Elsevier/North-Holland, 1976.

Akil, H., Mayer, D. J., \& Liebeskind, J. C. Antagonism of stimulation produced analgesia by naloxone, a narcotic antagonist. Science, 1975, 191, 961-962.

Awouters, F., Niemegeers, C. J. E., Lenaerts, F. M., \& Janssen, P. A. J. The effects of Suprofen in rats with Mycobacterium butyricum-induced arthritis. Arysneimittel-Forsch, 1975, 25, 1526-1537.

BALL, G. G. Electrical self-stimulation of the brain and sensory inhibition. Psychonomic Science, 1957, 8, 489-490.

Black, P., Cianci, S. N., \& Markowitz, R. F. Alleviation of pain by hypothalamic stimulation in the monkey. Confinia Neurologica, 1972, 34, 374-381.

Brady, J. V., \& Conrad, D. G. Some effects of limbic system self-stimulation upon conditioned emotional behavior. Journal of Comparative and Physiological Psychology, 1960, 53, 128 137.

Chance, W. T., White, A. C., Krynock, G. M., \& Rosecrans, J. A. Autoanalgesia: Behaviorally-activated antinociception. European Journal of Pharmacology, 1977, 44, 283-284.

Colpaert, F. C., De Witte, Ph., Maroli, A. N., Awouters, F., Niemegeers, C. J. E., \& Janssen, P. A. J. Self-administration of the analgesic Suprofen in arthritic rats: Evidence of Mycobacterium butyricum-induced arthritis as an experimental model of chronic pain. Life Sciences, 1980, 27, 921-928.

Cox, V. C., \& Valenstein, E. S. Attenuation of aversive properties of peripheral shock by hypothalamic stimulation. Science, 1965, 149, 323-325.

Dennis, S. G., Choiniere, M., \& Melzack, R. Stimulationproduced analgesia in rats: Assessment by two pain tests and correlation with self-stimulation. Experimental Neurology, 1980, 68, 295-309.

De WitTe, Ph. Compensatory effects of hypothalamic rewarding stimulation during concomitant painful footshock. Physiology \& Behavior, 1979, 22, 429-434.

Esposito, R. U., \& Kornetsky, C. Morphine lowering of selfstimulation thresholds: Lack of tolerance with long-term administration. Science, 1977, 195, 189-191.

Goldste IN, A. Opioid peptides in pituitary and brain. Science, 1976, 193, 1081-1086.

Liebman, J. M., Mayer, D. J., \& Liebeskind, J. C. Selfstimulation loci in the midbrain central grey matter of the rat. Behavioral Biology, 1973, 9, 299-306.

Maroli, A. N., Tsang, W. K., \& Stutz, R. M. Morphine and self-stimulation: Evidence for action on a common neural substrate. Pharmacology Biochemistry and Behavior, 1978, 8, 119-123.

McIntire, R. W. Conditioned suppression and self-stimulation. Psychonomic Science, 1966, 5, 273-274.

Melzack, R., \& Wall, P. D. Pain mechanisms: A new theory. A gate control system modulates sensory input from the skin before it evokes pain perception and response. Science, 1965, 150, 971-979.

Rose, D. M. Pain-reducing properties of rewarding electrical brain stimulation in the rat. Journal of Comparative and Physiological Psychology, 1974, 87, 607-617.

Stark, P., \& Fuller, R. W. Behavioral and biochemical effects of p-chlorophenylalanine, 3-chlorothyrosine and 3-chlorotyramine. A proposed mechanism for inhibition of self-stimulation. Neuropharmacology, 1972, 11, 261-272.

(Manuscript received November 29, 1982; revision accepted for publication June $22,1983$. ) 